\title{
RADIOLUMINESCENCE RESPONSE OF GERMANOSILICATE OPTICAL FIBRES
}

\author{
M R KHANLARY, P D TOWNSEND \\ MAPS, University of Sussex, Brighton, BN1 9QH, UK \\ and \\ J E TOWNSEND \\ ORC, University of Southampton, Southampton, SO9 5NH, UK.
}

\begin{abstract}
$\mathrm{X}$-ray irradiation of germanosilicate optical fibres simultaneously produces signals from both the core and substrate and so the radioluminescence spectra record the defect structure of both regions. The data provide evidence for the presence of dopants and trace impurities, as well as intrinsic defects formed by thermal and radiation processing. Examples of the changes in spectra or luminescence sensitivity with radiation dose, the influence of fibre pulling conditions and post irradiation heating are noted. The temperature dependence of the radioluminescence is reported. Whilst most of the intrinsic defects produce broad emission bands, rare earth dopants show line features. However, line features have also been noted for Al doped fibres. Such studies of fibre luminescence offer a sensitive monitor of changes in the structure of the glass network.
\end{abstract}




\section{INTRODUCTION,}

Among the most sensitive techniques for studying defects and trace impurities in insulators is to use X-ray induced luminescence. Whilst there has been considerable effort devoted to studies of imperfections in simple crystals, such as quartz, there has been rather less progress with amorphous material, even for silica, and very limited progress with the complex situation of optical fibres ${ }^{[1-6]}$. Not least among the problems is that the fibres comprise three regions. These are a central high index core, a lower index cladding and a thick supporting structure, the substrate. For penetrating radiation such as $\mathrm{X}$-rays all three regions are excited and hence luminescence is generated simultaneously from each. In view of the rather sparse literature ${ }^{[7-10]}$ it is fortunate that the defects in the germanosilicate cores and the silica based substrate material tend to be dominated by the familiar imperfections found in crystalline quartz and amorphous silica variations of $\mathrm{SiO}_{2}$. These generally involve broken oxygen bonds which act not only as electron traps but also as sites for forming complexes with impurities, new intrinsic linkages or structural relaxations. One must note however that radioluminescence is not a passive probe of the imperfections as X-rays can form new defects, modify existing ones and lead to changes in density and refractive index. Among the more familiar luminescence bands the signals near $460 \mathrm{~nm}$ are thought to arise from exciton decay and the $400 \mathrm{~nm}$ peak is clearly linked to the Ge sites, particularly in the core. Precise identification of sites involving electron-hole recombination is difficult as, although the ideal intrinsic relaxations of self trapped excitons (STE) are thought to occur near $460 \mathrm{~nm}(2.7 \mathrm{eV})$, there are a variety of similarly positioned emission bands in which electron-hole recombination occurs at defect sites which are only slightly perturbed relative to the perfect silica tetrahedra (e.g. defect sites of the $\mathrm{E}^{\prime}$ type, peroxy bonds and complexes). In principle there may be distinctly different emission bands where the transitions are located at specific impurities, (e.g. as for transitions within the energy levels scheme of rare earth ions). Variations in reported spectra also occur as a result of uncertainties in the experimental correction factors.

It should be noted that because the substrate is not part of the light guiding region it may not be as carefully prepared as the core, but it forms 
more than $90 \%$ of the optical fibre structure. Thus it might be assumed that luminescence signals from optical fibres will be dominated by the substrate, however this is not the case and in most of the data reported the luminescence efficiency of the core far exceeds that of the substrate. Radioluminescence (RL) is therefore a viable method to study differently prepared fibre cores.

\section{EXPERIMENTAL}

Various samples of pure silica, fibre preforms, drawn optical fibres and powdered fibre material have been examined. In the fibre examples the plastic cladding was chemically removed. The "pure" silica was Spectrosil B from Thermal Syndicate Ltd with total impurities quoted below $0.2 \mathrm{ppm}$. All fibres and preforms were prepared at Southampton University using previously described methods $[11,12]$. Table I lists the various preforms and fibres used in this or our earlier studies. Defects are introduced during the initial deposition of a porous frit (i.e. the precursor of the core and cladding), during chemical additions of laser dopants, during the thermal collapse of the frit and substrate tube into a preform, and finally during fibre drawing. Of the thermodynamic processes it is assumed that the final heating stage of fibre pulling is likely to be most critical as many of the earlier formed defects will anneal during the final heating phase. The melt temperature and drawing speed are therefore quoted. The list includes samples numbered ND297, ND324, ND326, ND414, ND416, ND424, ND578A and ND603A. (Note that only a selection of the data are discussed in this paper). All the preform samples have the same substrate material but differ in the additives in the core and cladding. ND414 has $P$ and $\mathrm{F}$ ions in the cladding and $\mathrm{Ge}, \mathrm{Er}$ and $\mathrm{Yb}$ in the core. ND416 has a pure silica cladding interface region but $\mathrm{Ge}$, and $\mathrm{Nd}$ in the core. Both ND414 and ND416 have a minimal amount of $\mathrm{Cl}$ added. The fibres are drawn with a standard "Heraelux WG" from Hereaus Silica and Metals Ltd tubing for the substrate. Only in two cases, ND324 and ND297 was $\mathrm{Cl}_{2}$ gas used in the drying stage after doping. Cladding regions normally contain $\mathrm{P}$ and $\mathrm{F}$ ions. Two aluminium doped preforms were fabricated, ND578A and ND603A, which contained 17.5 and 7 mole percent alumina in their cores, as determined from refractive index profile measurements. Both samples also included several ppm 
of $\mathrm{OH}$ ions. Finally, a sample $\mathrm{N} 9$ was prepared without a core but processed into fibres. This was done to compare effects of drawing speed and melt temperature on defects which are quenched into the substrate.

RL spectra were taken during excitation with a $30 \mathrm{kVp} \mathrm{X}$-rays with a tube current of $15 \mathrm{~mA}$ which delivers some $30 \mathrm{~Gy} / \mathrm{min}$ at the sample. Fresh samples were normally used for each set of measurements because, as will be mentioned later, the emission spectra may change after irradiation and heating cycles. Spectra were recorded with an $\mathrm{f} / 4$ scanning monochromator, pulse counting with a cooled red sensitive photomultiplier (S20 photocathode) and a small microcomputer data collection system. In all cases the spectra were corrected for the wavelength dependent efficiency of the system.

Temperature control was available either on a cryostat from 40 to $300 \mathrm{~K}$ or on a heater above room temperature from 20 to $400^{\circ} \mathrm{C}$.

\section{RESULTS and COMMENTS}

\subsection{Luminescence sensitivity}

Luminescence has variously been noted from preforms and fibres but, at least in terms of the spectra, they are quite similar although there are large differences in the rate of change of signal with radiation dose. In order to give a reference to the more familiar silica material, Figure 1a shows the spectra versus dose dependence for a silica sample during radioluminescence at room temperature. Figure $1 \mathrm{~b}$ gives an example of a wavelength slice, in this case the tenth. Note each scan requires some 30 seconds so the sample had received a dose of approximately $150 \mathrm{~Gy}$ by this stage. A comparable example of dose variations and wavelength slice is presented in figure 2 for fibre preform ND424. Data for the similarly prepared preform samples ND414 and ND416 are given in figures $3 \mathrm{a}$ and $3 \mathrm{~b}$. Data for the fibre version of ND414, together with examples of the fibres ND297 and ND324 are given in figures 4a, 4b and 4c. The differences between fibre and preform are quite subtle for example both versions of ND414 have similar spectra but for the ND414 fibre the blue emission envelope grows faster with $\mathrm{X}$-ray dose. Within experimental error the spectra of ND414 and ND416 are the same. Whilst the data show considerable similarity in the emission spectra they disguise the fact that the relative luminescence intensities differ significantly between these examples. The sample 
ND414 is much brighter than ND416 despite the similarity in spectra. ND414 is also brighter than ND324. It is significant however that ND414 is more heavily doped than either ND416 or ND324 ( see Table I).

A totally different spectral response was observed for the preforms of the Al doped samples ND578A and ND603A. The spectra are compared in figure 5 from which sees line type features superposed on a weaker blue emission band. The line features are stable over the dose range used but there is a small growth of blue emission as a result of the ionisation damage. Note that the more heavily doped sample (ND578A) has a stronger blue set of lines.

\subsection{Effects of heating.}

The dose dependence and spectra are further modified for samples which have been both irradiated and heated. In order to demonstrate such effects the same sample has been irradiated and $\mathrm{RL}$ data recorded, heated for thermoluminescence, and then a second RL growth curve obtained. Figures 6a and $6 \mathrm{~b}$ record the corresponding $\mathrm{RL}$ growth curves for the coreless fibre N9. Not surprisingly, it shows component bands more typical of pure silica than of the fibre examples, with relatively clear resolution of the component features near 400 and $460 \mathrm{~nm}$. Other bands near 350 and $530 \mathrm{~nm}$ are also indicated. However after heating to $400^{\circ} \mathrm{C}$ re-excitation of this substrate material gives a greatly modified initial spectrum and also a changed dose dependence of the components. In the initial excitation the 460 and $530 \mathrm{~nm}$ bands are of similar intensity and well resolved. With irradiation the $400 \mathrm{~nm}$ band grows slightly faster than the others. However after the heating cycle to $400^{\circ} \mathrm{C}$ the $460 \mathrm{~nm}$ band is initially twice as intense as the $400 \mathrm{~nm}$ band. However this intrinsic exciton band at $460 \mathrm{~nm}$ does not increase very rapidly with $\mathrm{X}$-ray dose whereas the $400 \mathrm{~nm}$ band, associated with $\mathrm{Ge}$, rises more quickly.

\subsection{Luminescence intensity and defect concentrations.}

Radiation sensitisation of luminescence is not unexpected for quartz, indeed, sensitisation of thermoluminescence signals by small radiation doses, and/or preheating, are routinely used in dating studies [13-16]. Similarly, both $\mathrm{RL}$ and $\mathrm{TL}$ effects have been noted in an earlier optical fibre study ${ }^{[17]}$ in which a green signal near $540 \mathrm{~nm}$ developed with a combination of radiation 
plus thermal treatment. In that example the $540 \mathrm{~nm}$ emission, as monitored by either $\mathrm{RL}$ or $\mathrm{TL}$, gave an indication of the total radiation history of the fibre.

At first sight the variations in luminescence efficiency are surprising as the substrate material is common throughout and the doped core represents perhaps only $5 \%$ of the total fibre cross section. Nevertheless the addition of impurity dopants and/or intrinsic defects in the core has changed the overall efficiency of light production, even though the same basic recombination centres appear to exist in most of the fibres. Except that the purer materials have stronger red emission bands. Intrinsic defect features can be influenced by rapidly cooling material from the melt, as during fibre drawing. Such processing amplified the emission intensity of the ND414 preform to fibre example. A combination of impurity and quenched in defects may thus be assumed to give a brighter signal from ND297 and ND324 since (see Table I) ND297 has some 7 times as many rare earth dopants in the core and was both pulled from a higher temperature and at a faster rate than ND324. Indeed the observation is that ND297 is relatively bright compared with ND324.

The role of the core in the overall luminescence signal is further exemplified by the study of the $\mathrm{Al}$ doped examples ND578A and ND603A. Figure 5 showed that in addition to the blue emission near $400 \mathrm{~nm}$ there are numerous strong line features. Their intensity, relative to the blue band, is similar for both the 7 and 17.5 mole \% alumina doping. At low temperature (77K) similar component features are apparent but their relative intensities are altered. Line features are unexpected from $\mathrm{Al}$ impurities but addition of trivalent $\mathrm{Al}$ at these high dopant levels may be accompanied by ppm traces of rare earth dopants which are also trivalent. Obvious candidates are Er and $\mathrm{Yb}$ as these have transitions near 480 and $540 \mathrm{~nm}$. The smaller line features at $630,590,440$ and 390 might correspond to other transitions from Tm, Ho, Er and $\mathrm{Nd}$ respectively. Note that for the present experimental conditions the line widths are defined by the steps and bandwidth of the scanning monochromator, so more precise identification is not realistic here. 


\subsection{Temperature dependence of luminescence.}

Quartz and silica differ further in the temperature dependence of the luminescence intensity with the ordered lattice giving a cleanly defined thermally activated change in intensity near $180 \mathrm{~K}[18,19]$ whereas the amorphous material has a much less clearly defined stage extending over a wider temperature range, as expected for a range of locally distorted silicate sites. In the spectrally resolved data of this RL study it is apparent that the various defect sites at which luminescence occurs have separate temperature dependencies. Further, the different fibres do not respond in an identical manner even though the emission may be built up from the same set of emission bands. The data are sufficiently complex in terms of band overlap and temperature sensitivity that detailed resolution of the component features is difficult and, without a clear need for the data, not currently valuable, except to emphasise the variation between the fibre types. Therefore a simple assessment of the competing processes has been judged by determining the effective activation energies in different temperature ranges. Activation energies for the temperature dependencies at the various emission band wavelengths are obtained by plotting the data as $\ln$ (intensity) versus $1 / \mathrm{T}$. Distinctly different values are apparent for the position of the band peaks, and even for a single sample, the activation energies are a function of wavelength, temperature range and type of silica, preform or fibre. Table II summarises values obtained for some of the specimens studied. Error estimates in the activation energy data are typically $\pm 0.01 \mathrm{eV}$ for the higher temperature range and $\pm 0.001 \mathrm{eV}$ at the lower temperatures.

The presence of two distinguishable activation energies at a single wavelength implies that there may be several mechanisms for transporting energy to a recombination centre. For example, the $460 \mathrm{~nm}$ emission is ascribed to an exciton relaxation luminescence, but this same emission could variously be generated by exciton diffusion or by hole or electron motion to a trapped charge. In either case the emission would appear as the characteristic electron-hole recombination luminescence as expected for an exciton. Note one cannot distinguish alternative pathways through the higher energy states in the current RL study. 


\section{DISCUSSION}

In terms of the spectral content the RL emission spectra of germanosilicate optical fibres and preforms closely resemble one another for a particular fibre core composition. Despite the substrate being by volume the major part of these optical fibres, the signals from the core dominate the spectra. The general trend is that the radiative decay paths of the absorbed energy is greatest in those fibre cores which contain the most imperfections, whether they be from dopants added during fibre fabrication, or defects induced by thermal and/or radiation. Consequently the spectra, the luminescence efficiency and the temperature dependencies of the various emission features change with core and history of the sample. Nevertheless the basic radiative defect sites are generally ascribable to the standard defects found in systems such as quartz and silica which are based on silicate tetrahedra. This similarity implies that to first order the luminescence sites are primarily at isolated point defects. The luminescence bands are typically signals from exciton decay (e.g. near $460 \mathrm{~nm})^{[20,21]}$, Ge impurities in the core (at $400 \mathrm{~nm})^{[10,22,23]}$, or from defects related to the $E^{\prime}$ type structures (e.g. near 380 to $390 \mathrm{~nm}$ and near 580 to $590 \mathrm{~nm})^{[24-26]}$. In other cases specific impurities such are rare earth ions show characteristic emission lines and, as with quartz features near 380 and $470 \mathrm{~nm}$ may relate to $\mathrm{H}_{3} \mathrm{O}_{4}$ and $\mathrm{AlO}_{4}$ impurities $[27,28]$. In practice, transitions between excited and ground states are not localized within a single silicate tetrahedron or at a single $\mathrm{Si}-\mathrm{O}$ bond, but instead are sensitive to neighbouring shells of ions. Inevitably with a wealth of potential defect sites, and the imprecision of local environmental structures around point defects which occur in a multicomponent glass network, the resultant RL signals are a complicated admixture of overlapping emission bands which are poorly resolved in either wavelength, temperature or activation energy as a result of distortions of the basic defect sites. The RL is a sensitive monitor of these distortions and the total concentration of imperfections, hence it is a convenient method of assessing them.

Although not discussed in detail above, a further observation is that the purer and more perfect materials emit more strongly in the red end of the spectrum. Slightly less obvious is that the purer samples show more clearly resolved features in the envelope of bands at the blue region. 


\section{CONCLUSION}

The radioluminescence signals from optical fibres provide a sensitive probe of the total defect concentration in the cores of the fibres. It may thus be used to analyse changes induced by different fabrication procedures.

\section{ACKNOWLEDGEMENTS}

We are grateful to the Iranian Ministry of Culture and Higher Education and the Optical Research Centre in Southampton for financial support.

\section{REFERENCES}

1. Alonso. P.J., Halliburton, L.E., Kohnke, E.E. and Bossoli, R.B., 1983, J. Appl. Phys, 54, 5369-5375.

2. Grinfelds, A.U., Aboltyn, D.E. and Plekhanov, V.G., 1984, Sov. Phys. Solid State, 26, 1075-1077.

3. Trukhin, A.N. and Plaudis, A.E., 1979, Sov. Phys. Sol. State, 21, 644-646.

4. Tanimura, K. and Halliburton, L.E., 1986, Phys. Rev. B34, 2933-2935.

5. Itoh, C., Tanimura, K. and Itoh, N., 1988, J. Phys, C21, 4693-4702.

6. Griscom, D.L., 1985, J. Non-Cryst. Solids, 73, 51-57.

7. Sigel, G.H. and Marrone,M.J., J. Non-Cryst. Solids, 45, 235-247 1981.

8. Kaiser,P., J. Opt. Soc. Amer., 64, 475,1974.

9. Friebele,E.J., Ginther, R.J. and Sigel, G.H., J. Appl. Phys.Letts, 24, 412-414, 1974.

10. Khanlary, M.R., Townsend, P.D. and Townsend, J.E., submitted for publication.

11. Nagel, S.R., MacChesney, J.B. and Walker,K.L., 1982, IEEE J. Quantum Electronics, QE15, 459-476.

12. Townsend, J.E., Poole, S.B. and Payne, D.N., 1987, Elect. Letts, 23, 329-331.

13. Horowitz, Y.S., 1984, Thermoluminescence and Thermoluminescence Dosimetry, CRC Press Inc, Boca Raton, USA.

14. Aitken, M.J., 1985, Thermoluminescence dating, Academic Press, London. 
15. Jani, M.G., Bossoli, R.B., and Halliburton, L.E., Phys. Rev B 27, 2285-2293, 1983.

16. McKeever, S.W.S., Strain, J.A., Townsend, P.D. and Uvdal, P., PACT 9, 123-132, 1983.

17. Ellis, A.E., Moskowitz,P.D., Townsend,J.E. and Townsend, P.D., 1989, J.Phys. D, 22, 1758-62.

18. Mattern, P.L., Lengweiler, K. and Levy, P.W., 1975, Rad Effects, 26, 237-248.

19. Malik, D.M., Kohnke, E.E. and Sibley, W.A., 1981, J. Appl. Phys., 52, 3600-3605

20. Itoh, C., Tanimura, K. and Itoh, N., 1989, Phys Rev. B39, 11183-11186.

21. Tanimura, K., Itoh, C. and Itoh, H., J. Phys C., 21, 1869-76, 1988.

22. Skuja, S.N. and Trukhin, A.N., 1989, Phys. Rev B39, 3090-3911.

23. Turner, W.H. and Lee, H.A., 1965, J. Chem. Phys, 43, 1428-1429.

24. Friebele,E.J., 1979, Optical Engineering, 18, 552-561.

25. Tsai, T.E., Griscom, D.L. and Friebele, E.J., 1987, Diffusion and Defect Data, 53/54, 469-476.

26. Luff, B.J. and Townsend, P.D., J. Phys C, 2, 8089-8097, 1990.

27. Yang, X.H. and McKeever, S.W.S., 1988, Nucl. Tracks Rad. Meas., 14, $75-9$.

28. Yang, X.H. and McKeever, S.W.S., 1990, J. Phys. D, 23, 237-244; ibid, 1990, Radiat. Protection Dosimetry, 33, 27-30. 


\section{FIGURE CAPTIONS}

1. (a) An isometric plot of the radioluminescence intensity versus wavelength and dose for a sample of Spectrosil silica. (b) A wavelength slice taken for the 10th scan.

2. (a) Isometric RL data for the dose dependence of a fibre preform type ND424. (b) A wavelength slice from the 10th scan.

3. Isometric RL data for the dose dependence for two fibre preforms (a) type ND414 and (b) ND416.

4. Isometric RL data for the dose dependence of fibres pulled from preforms type (a) ND414, (b) ND297 and (c) ND324.

5. A comparison of RL spectra from Al doped fibres ND578A and ND603A taken at room temperature.

6. Data taken with a fibre drawn from only the substrate material in which the initial RL data from sample N9 are shown in curve (a) however, as shown by (b) the growth curves are significantly altered after the sample had been heated to $400^{\circ} \mathrm{C}$. 
(a)
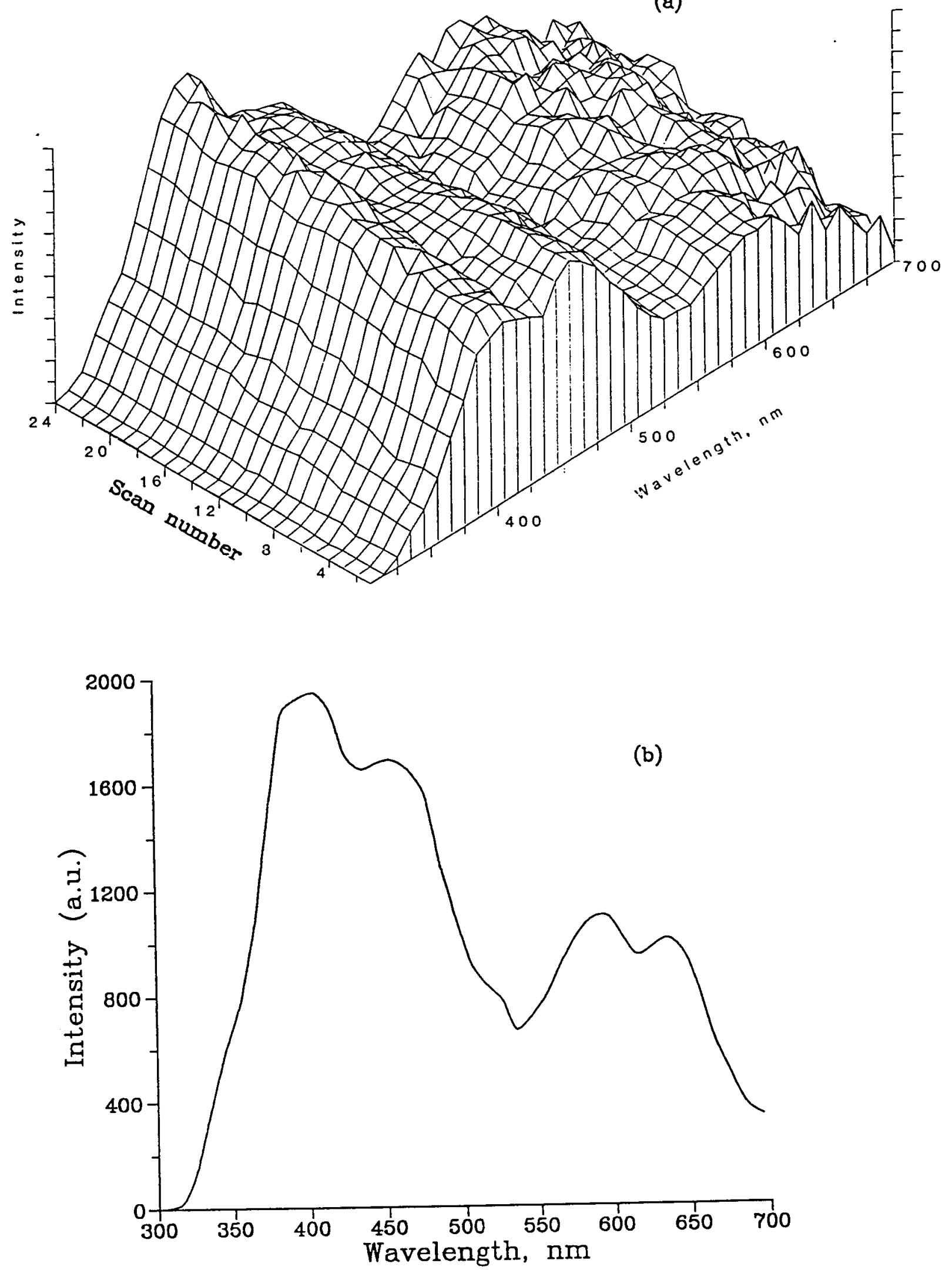

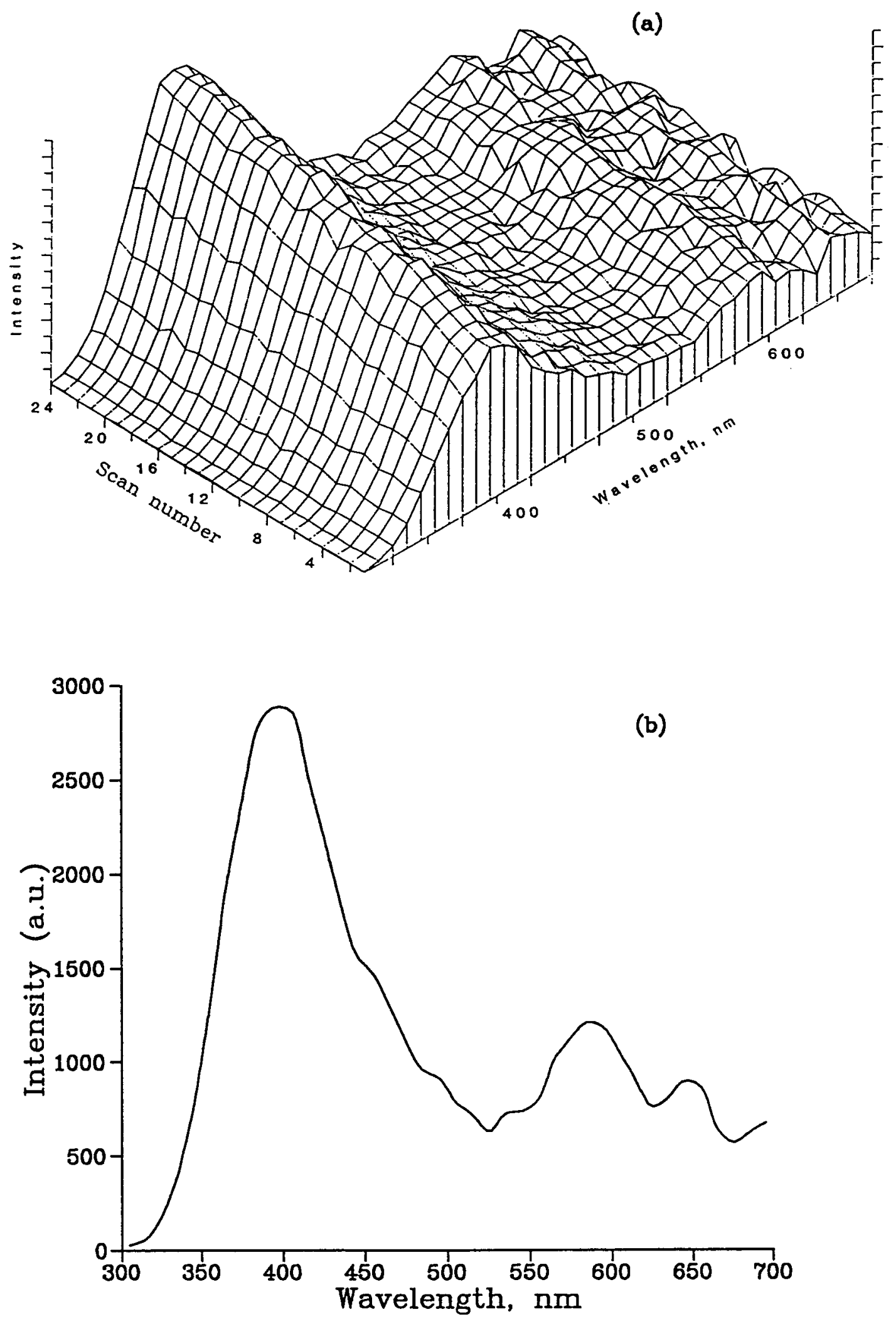

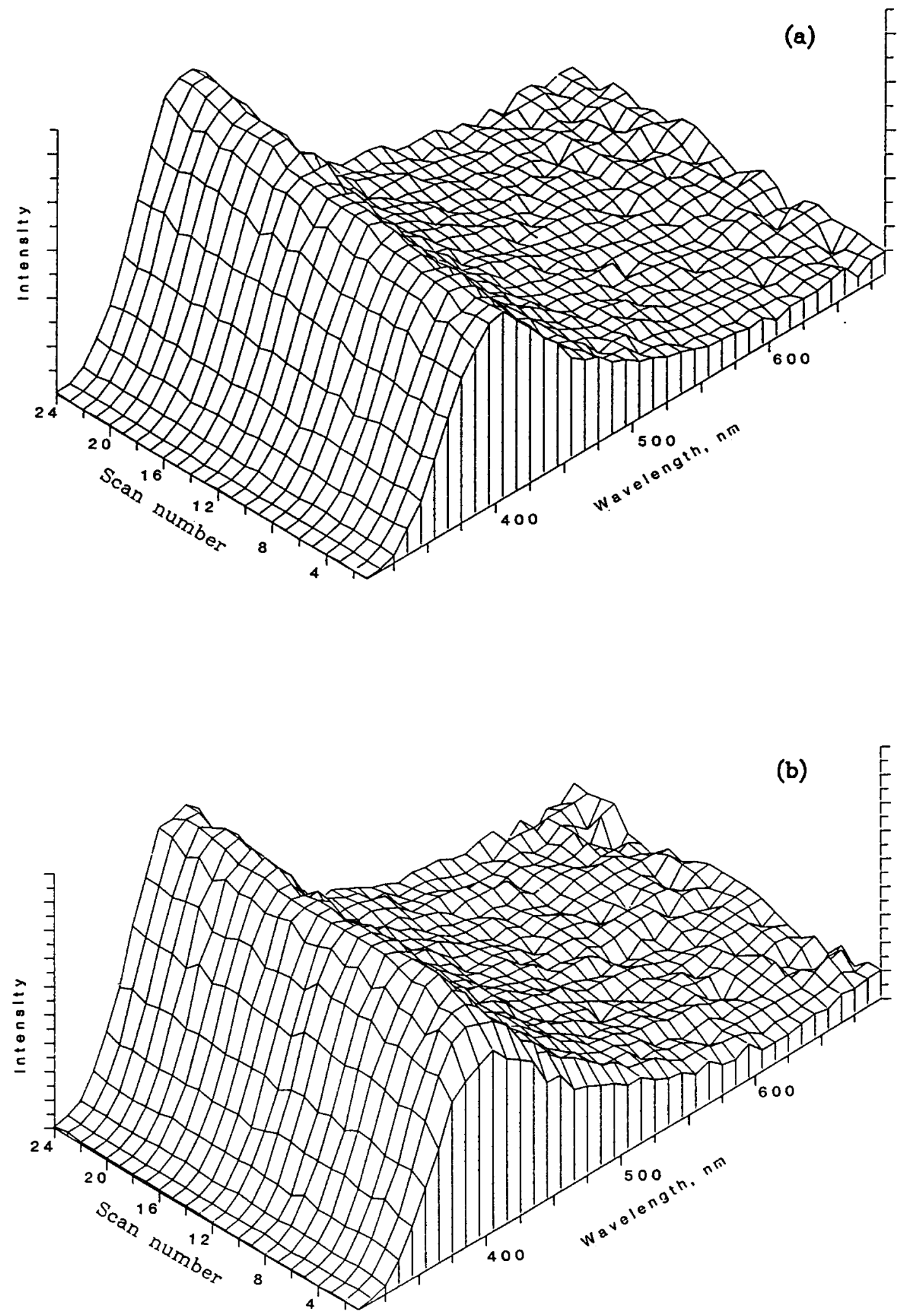


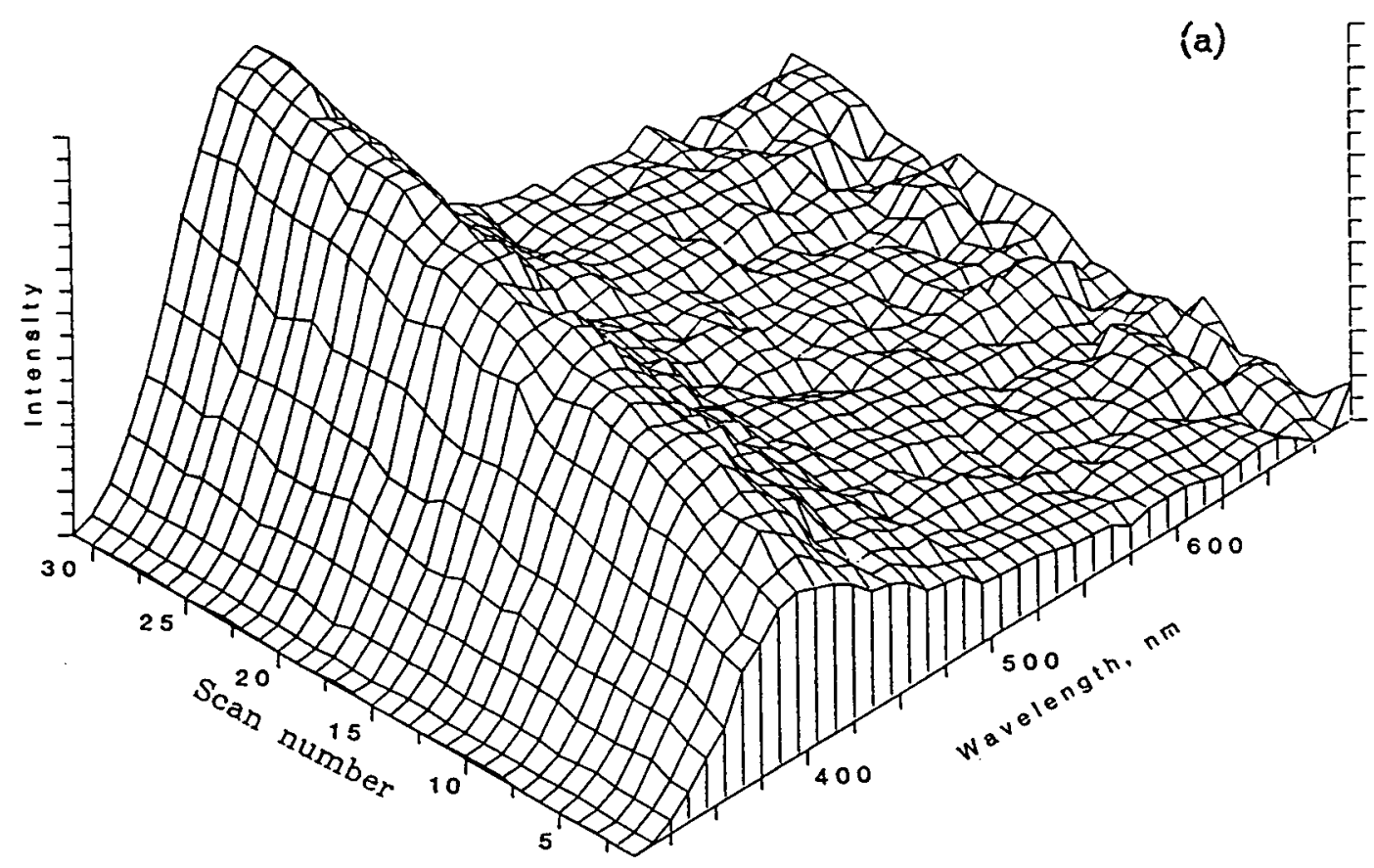

(b)

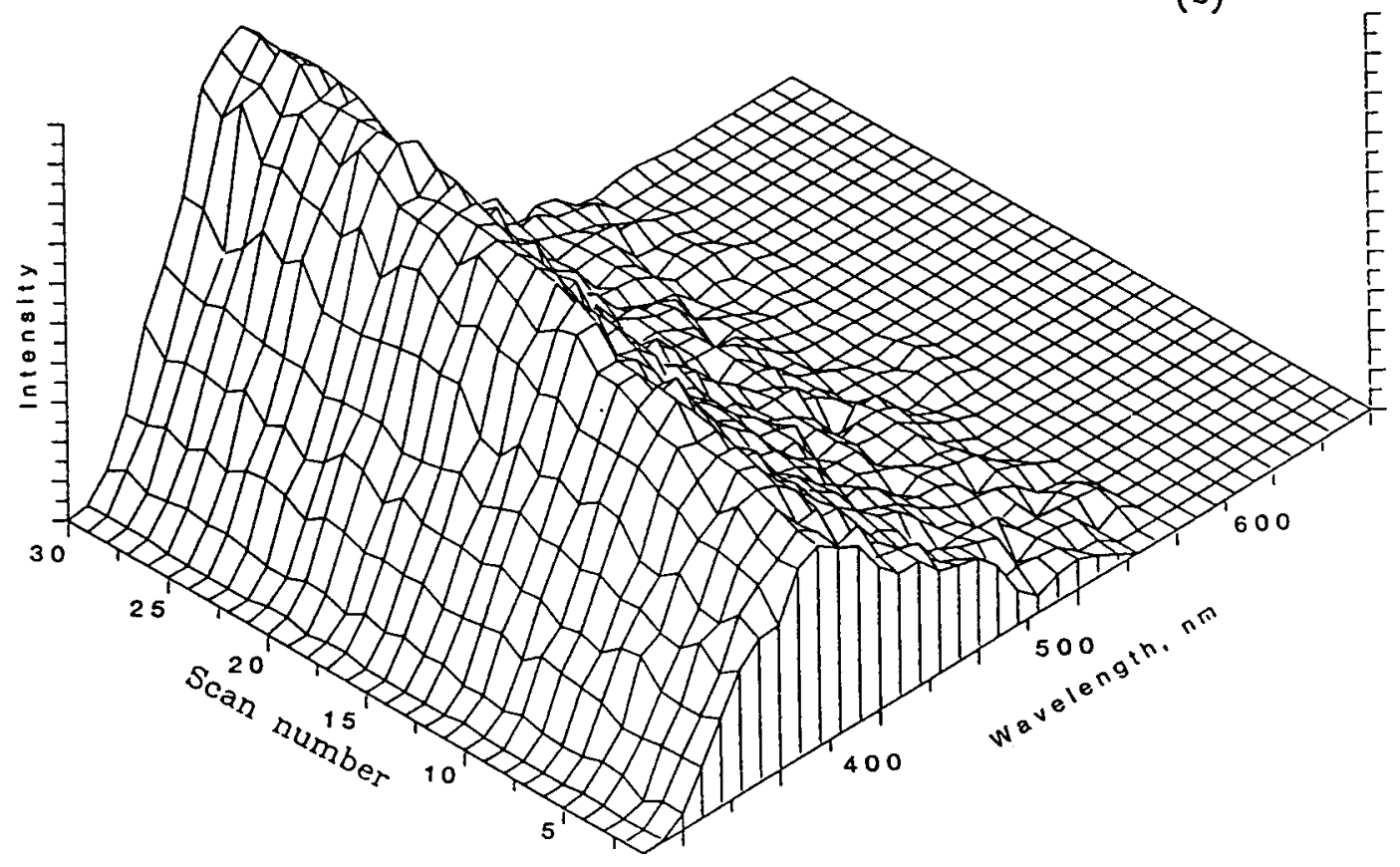




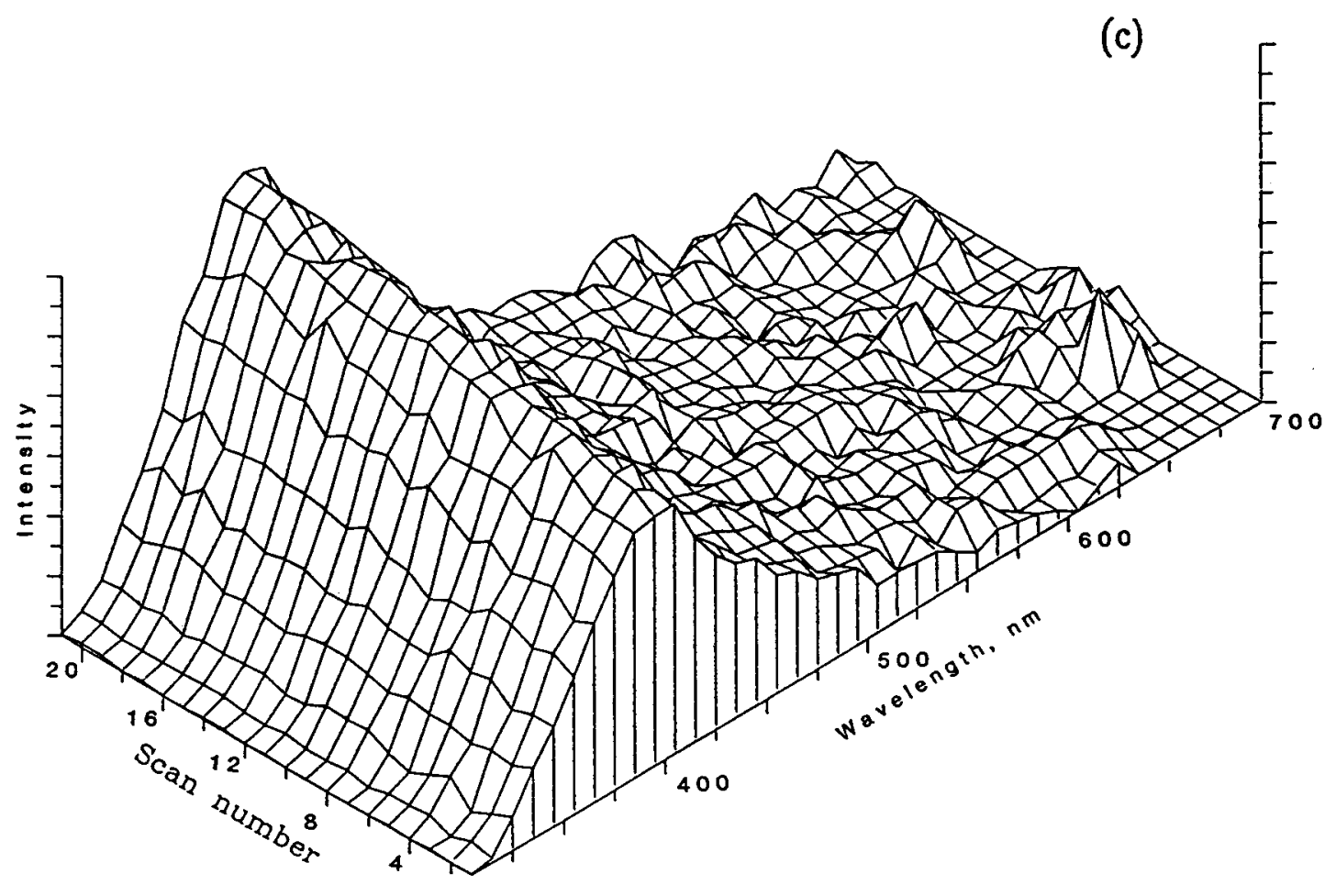




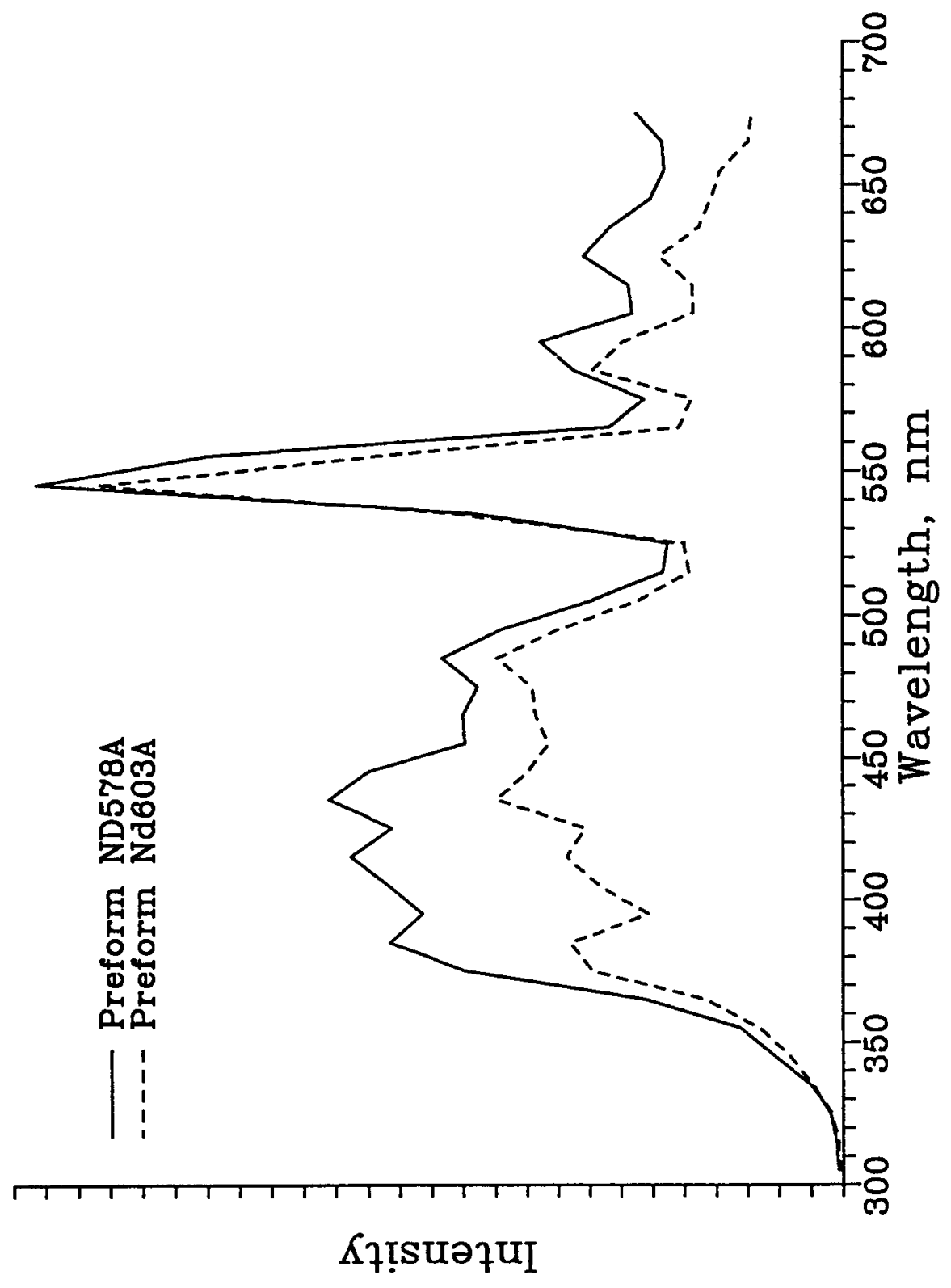


(a)
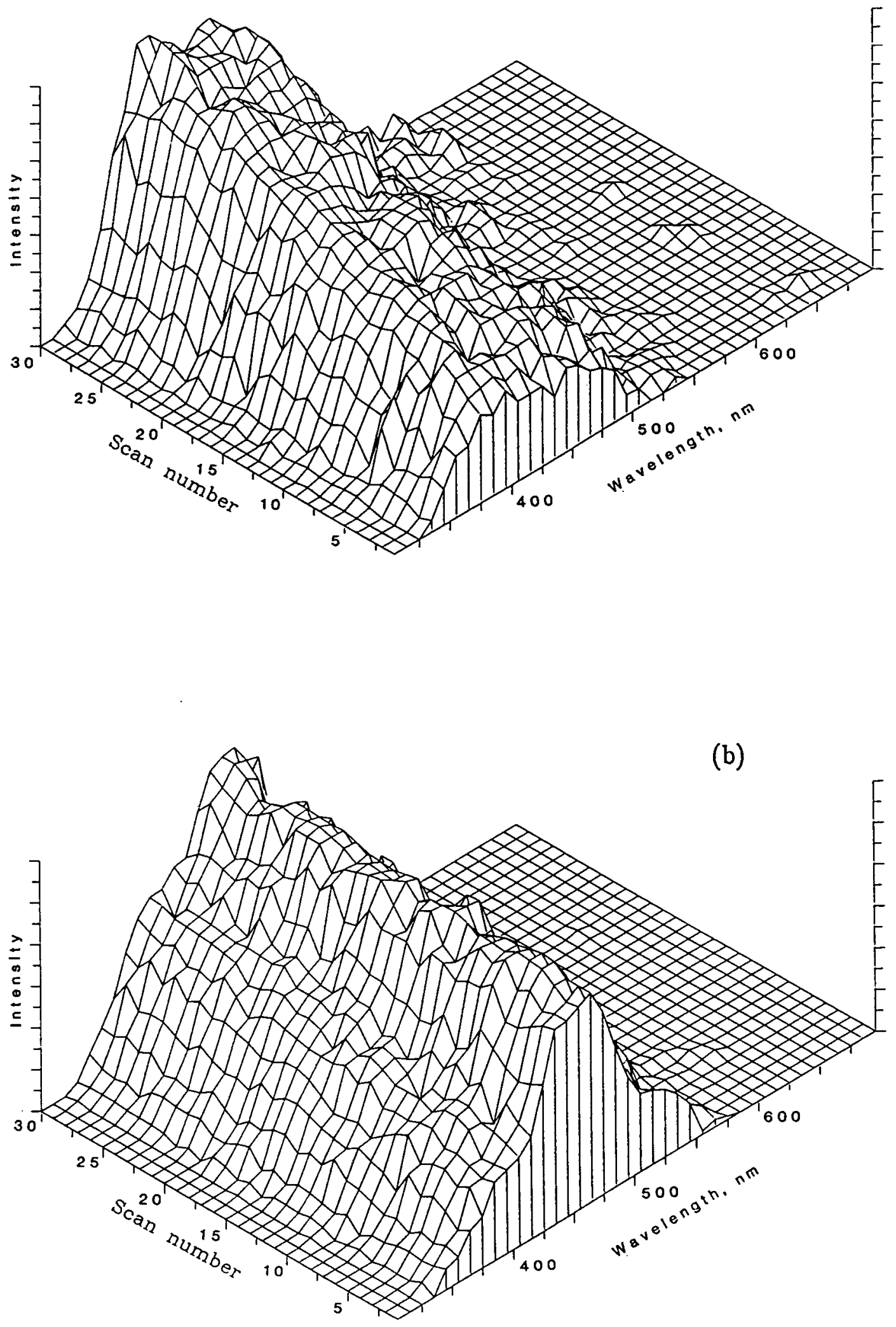
Table I

\begin{tabular}{|c|c|c|c|c|c|}
\hline $\begin{array}{c}\text { Fibre } \\
\text { Number }\end{array}$ & \multicolumn{2}{|c|}{ Core Dopant * } & $\begin{array}{c}\text { Pulling } \\
\text { Temperature }\left({ }^{\circ} \mathrm{C}\right) \\
\end{array}$ & $\begin{array}{c}\begin{array}{c}\text { Pulling } \\
\text { (rpm) } \dagger\end{array} \\
\end{array}$ & $\begin{array}{l}\text { Speed } \\
\mathrm{m} / \mathrm{min}\end{array}$ \\
\hline ND297-04 & \multicolumn{2}{|c|}{$\mathrm{Nd} ; 1400 \mathrm{ppm}$} & 2150 & 550 & 31 \\
\hline ND324-01 & \multicolumn{2}{|c|}{ Ho; 200 ppm } & 2097 & 400 & 22.6 \\
\hline ND326-01 & \multicolumn{2}{|c|}{ Nd; 225 ppm } & 2065 & 380 & 21.5 \\
\hline ND414-01 & \multicolumn{2}{|c|}{$\begin{array}{l}\mathrm{Er} ; 1100 \mathrm{ppm} \\
\mathrm{Yb} ; 4500 \mathrm{ppm}\end{array}$} & 2100 & 300 & 17 \\
\hline Substrate N9 & \multicolumn{2}{|c|}{ - } & 2108 & 480 & 27 \\
\hline $\begin{array}{l}\text { preform } \\
\text { Number }\end{array}$ & Core & Cladding & - & \multicolumn{2}{|c|}{-} \\
\hline ND414 & $\mathrm{Er}, \mathrm{Yb}$ & $P, F$ & - & \multicolumn{2}{|c|}{-} \\
\hline ND416 & $\mathrm{Nd}$ & - & - & \multicolumn{2}{|c|}{-} \\
\hline $\mathrm{Nd} 424$ & - & - & - & \multicolumn{2}{|c|}{-} \\
\hline ND578A & $\begin{array}{c}17.5 \% \\
\mathrm{Al}\end{array}$ & - & - & \multicolumn{2}{|c|}{-} \\
\hline ND603A & $7 \% \mathrm{Al}$ & - & - & \multicolumn{2}{|c|}{ - } \\
\hline
\end{tabular}

* All of the samples have $\mathrm{Ge} / \mathrm{Si}$ in the core and $\mathrm{Si}$ in the cladding.

$\dagger$ Drum diameter is about 18 millimeters. 
Table II Activation energy (E) of RL peaks in X-irradiated silica and optical fibre at different wavelength.

\begin{tabular}{|c|c|c|c|}
\hline Sample & $\begin{array}{c}\text { Wavelength, } \\
n \mathrm{~m}\end{array}$ & $\mathrm{~T}^{\prime}$ range $\left({ }^{\circ} \mathrm{K}\right)$ & $E(e v)$ \\
\hline \multirow{3}{*}{ Pure silica } & 460 & $\begin{array}{c}70-160 \\
160-280\end{array}$ & $\begin{array}{c}0.0026 \\
0.047\end{array}$ \\
\hline & 590 & $\begin{array}{c}70-120 \\
120-280\end{array}$ & $\begin{array}{c}0.0056 \\
0.015\end{array}$ \\
\hline & 640 & $70-280$ & 0.009 \\
\hline \multirow{3}{*}{$\begin{array}{c}\text { Fibre } \\
\text { preform } \\
\text { ND } 416\end{array}$} & 475 & $150-240$ & 0.016 \\
\hline & 570 & $70-160$ & 0.01 \\
\hline & 640 & $80-160$ & 0.017 \\
\hline \multirow{2}{*}{$\begin{array}{c}\text { Fibre } \\
\text { preform } \\
\text { ND } 414\end{array}$} & 450 & $\begin{array}{c}70-150 \\
150-240\end{array}$ & $\begin{array}{l}0.001 \\
0.026\end{array}$ \\
\hline & 590 & $70-170$ & 0.006 \\
\hline \multirow{3}{*}{$\begin{array}{l}\text { Fibre } \\
\text { ND } 414\end{array}$} & 450 & $\begin{array}{c}70-160 \\
160-280\end{array}$ & $\begin{array}{l}0.009 \\
0.037\end{array}$ \\
\hline & 520 & $\begin{array}{c}70-160 \\
160-250\end{array}$ & $\begin{array}{l}0.003 \\
0.055\end{array}$ \\
\hline & 590 & $80-250$ & 0.013 \\
\hline
\end{tabular}

